Discrete Comput Geom 26:269-281 (2001)

DOI: $10.1007 / \mathrm{s} 00454-001-0038-7$

\title{
Locked and Unlocked Polygonal Chains in Three Dimensions*
}

\author{
T. Biedl, ${ }^{1}$ E. Demaine, ${ }^{1}$ M. Demaine, ${ }^{1}$ S. Lazard, ${ }^{2}$ A. Lubiw, ${ }^{1}$ J. O'Rourke, ${ }^{3}$ \\ M. Overmars, ${ }^{4}$ S. Robbins, ${ }^{5}$ I. Streinu, ${ }^{3}$ G. Toussaint, ${ }^{5}$ and S. Whitesides ${ }^{5}$ \\ ${ }^{1}$ Department of Mathematics, University of Waterloo, \\ Waterloo, Ontario, Canada N2L 3G1 \\ \{biedl, eddemaine, mldemaine, alubiw\}@uwaterloo.ca \\ ${ }^{2}$ INRIA Lorraine, \\ Villers-les-Nancy Cedex 54602, France \\ lazard@loria.fr \\ ${ }^{3}$ Department of Computer Science, Smith College, \\ Northampton, MA 01063, USA \\ \{orourke, streinu\}@cs.smith.edu \\ ${ }^{4}$ Department of Computer Science, Utrecht University, \\ 3508 TB Utrecht, The Netherlands \\ markov@cs.ruu.nl \\ ${ }^{5}$ School of Computer Science, McGill University, \\ Montreal, Quebec, Canada H3A 2K6 \\ \{stever, godfried, sue\}@cs.mcgill.ca
}

\begin{abstract}
This paper studies movements of polygonal chains in three dimensions whose links are not allowed to cross or change length. Our main result is an algorithmic proof that any simple closed chain that initially takes the form of a planar polygon can be made convex in three dimensions. Other results include an algorithm for straightening open chains having a simple orthogonal projection onto some plane, and an algorithm for making convex any open chain initially configured on the surface of a polytope. All our algorithms require only $O(n)$ basic "moves."
\end{abstract}

* This research was initiated at a workshop at the Bellairs Research Institute of McGill University, Jan. 31-Feb. 6, 1998, organized by A. Lubiw and S. Whitesides. This is a revised and expanded version of abstract $\left[\mathrm{BDD}^{+} 1\right]$. Research supported in part by FCAR, NSERC, and NSF. 


\section{Introduction}

A polygonal chain $P=\left(v_{0}, v_{1}, \ldots, v_{n-1}\right)$ is a sequence of consecutively joined segments $e_{i}=v_{i} v_{i+1}$ (also called edges or links) of fixed lengths $\ell_{i}=\left|e_{i}\right|$, embedded in space. ${ }^{1}$ A chain is closed if $v_{n-1}=v_{0}$; otherwise, it is open. A closed chain is also called a polygon. A chain is simple if its edges are pairwise disjoint except for adjacent edges, which share only their common endpoint. Unless stated otherwise, chain means "simple polygonal chain." For an open chain, our goal is to straighten it; for a closed chain the goal is to convexify it, i.e., to reconfigure it to a planar convex polygon. Both goals are to be achieved by continuous motions that maintain link lengths and simplicity of the chain throughout.

A locked chain is one that cannot be straightened or convexified. Since a chain in three dimensions can be continuously moved between any of its unlocked configurations via straightened or convexified intermediate configurations, the property of being unlocked is of fundamental importance. Nontrivial knots provide examples of closed chains that are locked. However, as Figs. 1 and 2 show, even open chains and unknotted closed chains may be locked in three dimensions. Section 2 provides details.

In Section 3 we give an algorithmic proof that any open chain with a simple orthogonal projection can be made straight in three dimensions, as well as an algorithmic proof that any open chain embedded in the surface of a polytope can be straightened. Section 4 presents our main result, an algorithmic proof that any closed chain initially taking the form of a polygon lying in the plane can be made convex.

We describe our algorithms in terms of "moves." Throughout the paper a "move" is a continuous motion of a chain in which only $O(1)$ angles at joints (vertices) change at once, and only $O(1)$ dihedral angles at edges change at once. (The dihedral angle of an edge is the angle between the plane it determines with one of its neighboring edges and the plane it determines with the other.) Our algorithms make easily described moves that change angles at a very small number of vertices and edges at once.

After we reported our work in abstract form $\left[\mathrm{BDD}^{+} 1\right]$, Connelly et al. [CDR] and Streinu $[\mathrm{St}]$ reported two approaches for convexifying polygons in the plane using

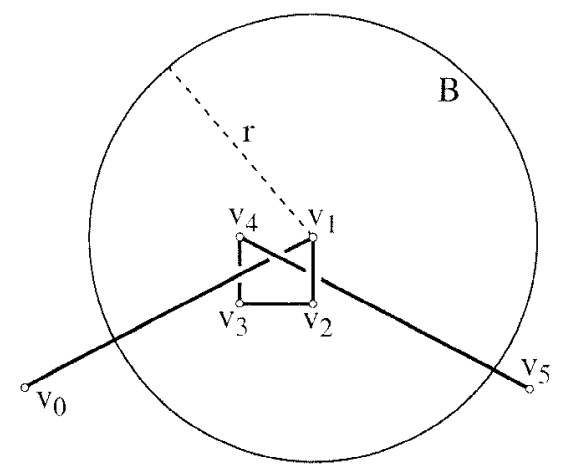

Fig. 1. A locked, open chain $K$ with long "knitting needles" at the ends.

\footnotetext{
${ }^{1}$ All index arithmetic throughout the paper is $\bmod n$.
} 


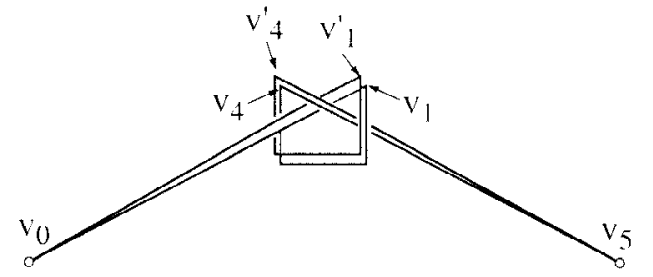

Fig. 2. $\quad K^{\prime}(K$ doubled): a locked but unknotted closed chain.

motions just in the plane. Our algorithm (Section 4) convexifies planar polygons using motions in three dimensions, which at first glance appears a less general result. However, the motions in all our algorithms are significantly simpler, consisting of a linear number of moves. (Our technical report $\left[\mathrm{BDD}^{+} 2\right]$ argues that the sequence of moves can be computed in $O(n)$ time on an extended real RAM model of computation.) On the other hand, the motions in [CDR] are described implicitly by the solution of a differential equation, and the motions in [St] are given by a high-degree algebraic curve. Both of these types of motions are difficult to compute and represent explicitly.

Background. The study of movement problems for polygonal chains goes at least as far back as Cauchy's 1813 theorem on the rigidity of polyhedra [Cr, Chapter 6]. Mechanical engineers have studied planar linkages, which exhibit a graph structure whose links are allowed to cross, since Peaucellier's 1864 linkage or earlier. Edge weighted graph embedding and reconfiguration problems, with or without a simplicity requirement, have arisen in many contexts, including molecular conformation $[\mathrm{CH}]$, mechanical design $[\mathrm{GN}],[\mathrm{Hu}],[\mathrm{Mc}]$, robotic animation [Ko], rigidity theory [Wh2], random walks [MS], and knot theory [A], [CJ], [Li], [Mi].

Basic questions concerning open and closed chains have proved surprisingly difficult. For example, the question of whether every planar, simple open chain can be straightened in the plane while maintaining simplicity went unsolved for several years. In piecewise linear knot theory, complete classification of the three-dimensional embeddings of closed chains with $n$ edges has been found to be difficult, even for $n=6[\mathrm{CJ}]$.

Any simple polygon lying in the plane may be made convex in three dimensions by "flipping" out the reflex pockets, i.e., rotating the pocket chain into three dimensions and back down to the plane; see Fig. 3. This simple procedure was suggested by Erdôs [Er] and proved to work by de Sz. Nagy [Na]. The number of flips, however, cannot be bounded as a function of the number of vertices $n$ of the polygon, as Joss and Shannon [Gr] first proved. See [To1] for the complex history of these results.

Previous computational geometry research on the reconfiguration of chains (e.g., [Ka], [KSW], [HJW2], [LW1], and [Wh1]) typically studies chains with crossing links, moving in two dimensions, sometimes in the presence of obstacles; Sallee [Sa] and Lenhart and Whitesides [LW2] study configurations of closed chains with crossing links in all dimensions $d \geq 2$. For more on weighted graph embedding and reconfiguration problems, see also, for example, [ELR ${ }^{+}$, [HJW1], [Ko], [CH], [Wh2], [SS], and [Ca]. In answer to an open problem we posed in a preliminary version $\left[\mathrm{BDD}^{+} 1\right]$ of this paper, Cocan and O'Rourke $[\mathrm{CO}]$ have shown that locked chains do not exist in dimensions greater than three. 


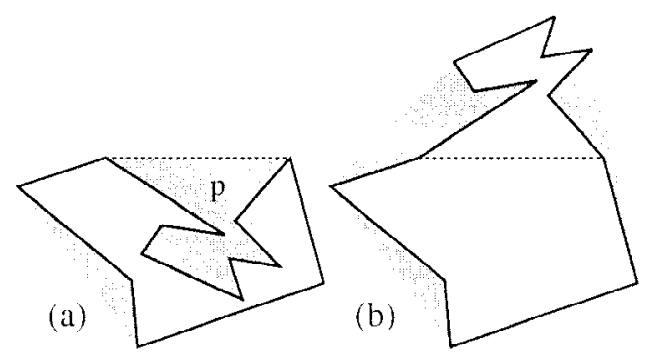

Fig. 3. (a) A pocket $p$. (b) The polygon after flipping $p$.

\section{Chains that Lock}

Here we discuss the examples shown in Figs. 1 and 2, which show that open chains and closed, unknotted chains may be locked. Both of these results were obtained independently by other researchers [CJ]. Our proofs use particularly simple constructions.

Consider the chain $K=\left(v_{0}, \ldots, v_{5}\right)$ configured as in Fig. 1, where the lengths $\ell_{0}$ and $\ell_{4}$ of the extreme edges are each much longer than the sum of the lengths of the intermediate edges. Let $B$ be a sphere centered at $v_{1}$ of radius $r$, where $\ell_{1}+\ell_{2}+\ell_{3}<r<$ $\ell_{4}-\left(\ell_{1}+\ell_{2}+\ell_{3}\right)$. Thus, no matter how the chain moves, $v_{2}, v_{3}$, and $v_{4}$ lie strictly inside $B$, whereas $v_{0}$ and $v_{5}$ lie strictly outside $B$. Join $v_{0}$ and $v_{5}$ outside $B$ with a long string to form a knot. The string should be long enough not to impede the motions of $v_{0}$ and $v_{5}$, and it should form an unknot if it were completed to a closed curve by the insertion of a straight string between $v_{0}$ and $v_{5}$. Alternatively, it is not difficult to design ways to join $v_{0}$ and $v_{5}$ with an unlocked chain $P$ consisting of a few long links that can track any motions of $v_{0}$ and $v_{5}$ while avoiding collisions. If chain $K$ could be straightened, then the knot $K \cup P$ could be made convex, a contradiction.

Next, "double" chain $K$ by adding vertices $v_{i}^{\prime}$ near $v_{i}$, for $1 \leq i \leq 4$, and connecting all these vertices to form a chain $K^{\prime}=\left(v_{0}, \ldots, v_{5}, v_{4}^{\prime}, \ldots, v_{1}^{\prime}\right)$. See Fig. 2. Any convexifying motion for $K^{\prime}$ brings $K$ to a configuration from which, removing the second copy of $K$, the first copy can be straightened, a contradiction. Thus $K^{\prime}$ is also locked. For examples of locked, unknotted polygons with only six edges, see [CJ] and [To2].

\section{Two Unlocked Families of Open Chains}

\subsection{Straightening Open Chains with Simple Projections}

Let $P$ be an open chain in three dimensions with a simple orthogonal projection $P^{\prime}$ onto the $x y$-coordinate plane, denoted $\Pi_{x y} .{ }^{2}$ This subsection describes an algorithm to

\footnotetext{
${ }^{2}$ Bose et al. [BGRT] provide a polynomial-time algorithm to determine whether $P$ admits a simple orthogonal projection onto a plane and to output a projection plane if it exists.
} 


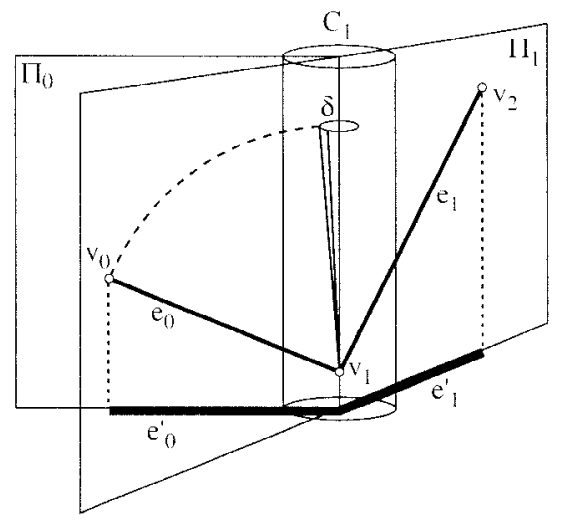

Fig. 4. Step 0 rotates $e_{0}$ in $\Pi_{0}$ about $v_{1}$ to bring $e_{0}$ into $C_{1}$; step 1 begins by rotating $e_{0}$ about the $C_{1}$ axis until $\Pi_{0}=\Pi_{1}$.

straighten $P$. In answer to open problem \#3 in our technical report [BDD ${ }^{+}$], Calvo et al. $\left[\mathrm{CKM}^{+}\right]$showed the more general result that any closed chain with a simple orthogonal projection $P^{\prime}$ on the plane can be convexified by using the method of Connelly et al. [CDR] as a subroutine. Our algorithm is much simpler.

We call lines and planes parallel to the $z$-axis "vertical," and in this subsection we label projections in $\Pi_{x y}$ with a prime. Let $P[i, j]$ denote the subchain $\left(v_{i}, v_{i+1}, \ldots, v_{j}\right)$, including endpoints $v_{i}$ and $v_{j}$. Let $P^{\prime}$ denote the projection of $P$ on $\Pi_{x y}$, let $d_{\min }$ denote the minimum distance between a vertex $v^{\prime}$ and a nonadjacent edge $e^{\prime}$, and choose a positive $r<d_{\min }$. Let $C_{i}$ denote a cylinder of radius $r$ with a vertical axis passing through $v_{i}$. Finally, let $\Pi_{i}$ denote the vertical plane containing $e_{i}$ and $e_{i}^{\prime}$.

For $0 \leq i<n$, the goal of step $i$ is to squeeze the links of $P[0, i+1]$ into $C_{i+1}$ so that $v_{0}$ lies within distance $(i+1) r / n$ of the axis of $C_{i+1}$, and so that $P[0, i+1]$ is monotone with respect to the line $\Pi_{i} \cap \Pi_{x y}$. By monotone, we mean that the intersection of the subchain with each vertical line in $C_{i+1}$ is either empty or a single point. Step $i=$ 0 achieves this goal by rotating $e_{0}$ in $\Pi_{0}$ about its endpoint $v_{1}$. See Fig. 4 .

To initialize for step $i+1$, subchain $P[0, i]$ is kept internally rigid and rotated as an object about the axis of $C_{i}$. In particular, $P[0, i]$ is rotated away from $e_{i}$ until $P[0, i]$ rotates into the vertical plane $\Pi_{i}$ of $e_{i}$. To achieve the goal configuration of step $i+1$, edge $e_{i}$ is rotated in $\Pi_{i}$ about its endpoint $v_{i+1}$ until its other endpoint, $v_{i}$, lies within distance $r / n$ of the axis of $C_{i+1}$; the angle between the edges incident to $v_{i}$ is changed so that any configuration of $P[0, i]$ during the rotation of $e_{i}$ is a translate of the configuration of $P[0, i]$ when the rotation of $e_{i}$ began. See Fig. 5 .

Once the entire chain has been moved to a planar, monotone configuration in $C_{n}$, the joints may be straightened one by one, working from one end of the chain to the other.

The algorithm performs $O(n)$ moves. To compute descriptions of these rotations on an extended real RAM requires computation of the cylinder radius $r$. This may be done in $O\left(n^{2}\right)$ time by computing each vertex-vertex distance and each edge-edge distance; in $\left[\mathrm{BDD}^{+} 2\right]$ we describe a method for computing $r$ in $O(n)$ time. 


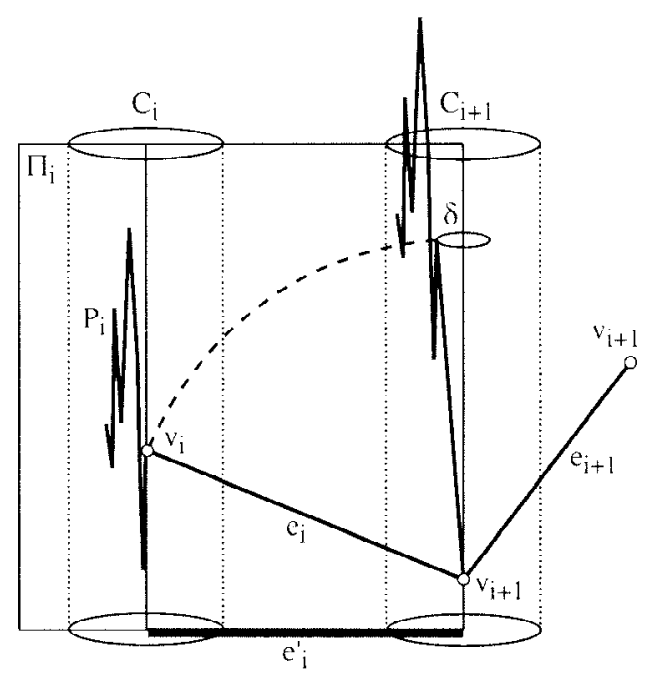

Fig. 5. Step $i+1$ rotates $e_{i}$ about $v_{i+1}$ in $\Pi_{i}$, dragging along $P_{i}$.

\subsection{Straightening Open Chains on a Polytope}

In this subsection we show that any open chain $P$ embedded on the surface of a convex polytope may be straightened. We start with the case of an open chain lying in $\Pi_{x y}$, which we straighten (essentially as in Section 3.1) as follows. Rotate $e_{0}$ about $v_{1}$ within $\Pi_{0}$ until $e_{0}$ is vertical; now $v_{0}$ projects into $v_{1}$ on $\Pi_{x y}$. In general, rotate $e_{i}$ about $v_{i+1}$ within $\Pi_{i}$ until $v_{i}$ sits over $v_{i+1}$. Throughout this motion, keep the previously straightened chain $P_{i}=P[0, i]$ above $v_{i}$ in a vertical ray through $v_{i}$.

An easy generalization of this "pick-up into a vertical ray" idea permits straightening any open chain lying on the surface of a convex polytope $\mathcal{P}$. The same procedure is followed, except that the surface of $\mathcal{P}$ plays the role of $\Pi_{x y}$, and surface normals play the roles of vertical rays. Suppose that a vertex $v_{i}$ of the polygonal chain $P$ lies on an edge or at a corner of $\mathcal{P}$, touching $d$ faces $f_{1}, \ldots, f_{d}$. Let $R_{1}, \ldots, R_{d}$ denote the rays through $v_{i}$ that are normal to $f_{1}, \ldots, f_{d}$, respectively. For each $v_{i}$, choose a preferred normal. When such a vertex $v_{i}$ is encountered, the line containing $P_{i}$ is rotated to the preferred normal for $v_{i}$.

This algorithm performs $O(n)$ rotations, and a description of these can be computed in $O(n)$ time on an extended real RAM. Note that it is possible to draw on a polytope surface a chain that has no simple orthogonal projection to a plane, so this algorithm handles some cases not covered by the algorithm of the previous subsection.

\section{Convexifying Planar Simple Polygons in Three Dimensions}

This section offers a new algorithm for convexifying planar closed chains. It is more complicated than the flipping method of Erdôs illustrated in Fig. 3 but has the advantage 
that it uses only a bounded number of moves, in fact $O(n)$ moves. Furthermore, in contrast to [CDR] and [St], our algorithm remains conceptually simple and the moves it performs are easily described.

We consider a closed chain $P$, initially in the form of a simple polygon in the $x y$-plane, $\Pi_{x y}$. We assume that $P$ has no more than two consecutive collinear vertices, as otherwise we can freeze and eliminate middle joints. For $\varepsilon>0$, let $\Pi_{\varepsilon}$ be the plane $z=\varepsilon$ parallel to $\Pi_{x y}$. Throughout this section primed labels indicate positions of vertices that have been lifted to $\Pi_{\varepsilon}$ or above, and $\Pi_{z}(p, q)$ denotes the vertical plane containing points $p$ and $q$. We say a subchain is convex if, taken together with the phantom segment joining its endpoints, it forms a convex polygon.

\subsection{Algorithm Overview}

The algorithm lifts vertices one by one out of $\Pi_{x y}$, accumulating them into a vertical convex "arch" $A$ with base vertices in $\Pi_{\varepsilon}$. The unlifted vertices remain fixed in their initial positions. Figure 6 shows an arch $A$ with base points $v_{0}^{\prime}$ and $v_{i}^{\prime}$ in $\Pi_{\varepsilon}$ (not shown). Arch $A$ lies above $\Pi_{\varepsilon}$ in a vertical plane $\Pi_{z}\left(v_{0}^{\prime}, v_{i}^{\prime}\right)$. Edges $v_{i}^{\prime} v_{i+1}$ and $v_{0}^{\prime} v_{n-1}$ connect the base points $v_{0}^{\prime}$ and $v_{i}^{\prime}$ of $A$ to $P[i+1, n-1]$ in $\Pi_{x y}$.

After a new vertex $v_{i+1}^{\prime}$ (and typically, its adjacent edge $v_{i}^{\prime} v_{i+1}^{\prime}$ ) is lifted to $\Pi_{\varepsilon}$, the arch is flipped down to $\Pi_{\varepsilon}$. There the arch together with the new vertex $v_{i+1}^{\prime}$ and its adjacent edge is reconvexified, with the future base points $v_{0}^{\prime}$ and $v_{i+1}^{\prime}$ held fixed during the process. The new arch is then flipped back up, rotating about the line through $v_{0}^{\prime} v_{i+1}^{\prime}$, to a new vertical plane $\Pi_{z}\left(v_{0}^{\prime}, v_{i+1}^{\prime}\right)$.

Performing reconvexification of the arch in $\Pi_{\varepsilon}$ prevents collisions between the links in the arch, the links remaining in the plane, and the two connecting links to the arch. Similarly, raising the arch to vertical while lifting a new vertex to $\Pi_{\varepsilon}$ prevents collisions between arch edges and connecting links.

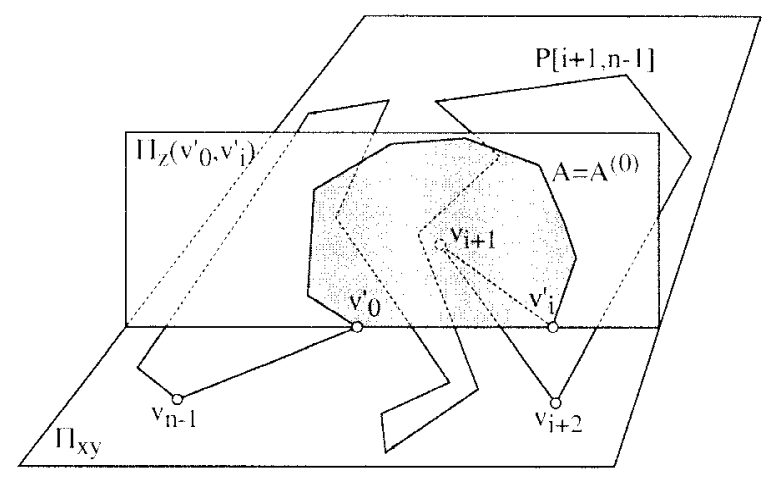

Fig. 6. The $\operatorname{arch} A$ after incorporating $P[0, i] ; v_{0}^{\prime}$ and $v_{i}^{\prime}$ lie in $\Pi_{\varepsilon}$ (not shown). 


\subsection{Procedures}

Our algorithm ${ }^{3}$ makes use of the following procedures $S_{0}-S_{4}$ : an initialization procedure $S_{0}$ that raises three vertices and creates a two-link arch; a vertex lifting procedure $S_{1}$, which lifts a new vertex $v_{i}$ to $\Pi_{\varepsilon}$; an arch lowering procedure $S_{2}$, which rotates the current arch $A$ about the line through its endpoints into $\Pi_{\varepsilon} ;$ a reconvexification procedure $S_{3}$, which convexifies the arch together with the vertex just lifted; and an arch raising procedure $S_{4}$, which returns the new arch to vertical.

At the end of the initialization $S_{0}$, the arch contains $v_{0}^{\prime}, v_{1}^{\prime}, v_{2}^{\prime}$. It then makes repeated passes through $S_{1}-S_{4}$. Here, for reference, are the hypotheses that are intended to hold at the beginning of the pass through $S_{1}-S_{4}$ that lifts $v_{i+1}$, for $2 \leq i \leq n-2$. (See Fig. 6.)

H1. Vertices $v_{0}^{\prime}$ and $v_{i}^{\prime}$ lie in $\Pi_{\varepsilon}$, and $v_{i+1}, \ldots, v_{n-1}$ lie in $\Pi_{x y}$ in their original positions.

H2. Subchain $P[0, i]$ has now been moved up to form a convex $\operatorname{arch} A$ in $\Pi_{z}\left(v_{0}^{\prime}, v_{i}^{\prime}\right)$; $A$ rises above $\Pi_{\varepsilon}$ and intersects $\Pi_{\varepsilon}$ only in $v_{0}^{\prime}$ and $v_{i}^{\prime}$.

H3. Points $v_{0}^{\prime}$ and $v_{i}^{\prime}$ project down to $\Pi_{x y}$ within distance $\delta$ of their original positions $v_{0}$ and $v_{i}$. (In Section 4.3 we compute $\delta>0$ from the initial configuration of $P$ such that at each vertex $v_{j}$, the disk of radius $\delta$ centered at $v_{j}$ includes no other vertices and intersects only the two edges incident to $v_{j}$, and such that any perturbed polygon obtained by displacing the vertices within their disks, ignoring the fixed link lengths, remains simple and without straight vertices.)

Next, we describe each of the procedures $S_{0}-S_{4}$ in detail, noting what constraints they impose on the choice of $\varepsilon$ and $\delta$ each time they are performed. Choosing $\varepsilon$ and $\delta$ that satisfy the constraints will enable us to prove that $\mathrm{H} 1-\mathrm{H} 3$ hold as intended. Later we indicate how to compute specific values for $\varepsilon$ and $\delta$ that satisfy all the constraints.

Note that procedures $S_{1}-S_{4}$ below do not apply until arch $A$ contains at least three vertices.

$S_{0}$ : initialization. Select an arbitrary (strictly) convex vertex $v_{1}$, and lift $v_{0}, v_{1}, v_{2}$ as follows. Rotate $v_{1}$ about the line through $v_{0} v_{2}$ up to $\Pi_{\varepsilon}$, and call its new position $v_{1}^{\prime \prime}$; rotate $v_{0}$ about the line through $v_{n-1} v_{1}^{\prime \prime}$ up to $\Pi_{\varepsilon}$, and call its new position $v_{0}^{\prime}$; rotate $v_{2}$ about the line through $v_{1}^{\prime \prime} v_{3}$ up to $\Pi_{\varepsilon}$, and call its new position $v_{2}^{\prime}$; finally, rotate $v_{1}^{\prime \prime}$ about the line through $v_{0}^{\prime} v_{2}^{\prime}$ upwards until it lies in the plane $\Pi_{z}\left(v_{0}^{\prime}, v_{2}^{\prime}\right)$, and let $v_{1}^{\prime}$ denote its new position above $\Pi_{\varepsilon}$. This last rotation rotates a triangle from a horizontal to a vertical plane to create the initial arch $A$, so the triangle must be nondegenerate.

$S_{0}$-constraints: during the initialization procedure, vertices $v_{0}, v_{1}, v_{2}$ must reach $\Pi_{\varepsilon}$, and $v_{1}^{\prime \prime}$ should not become straight (to prevent a degenerate arch).

$S_{1}$ : lifting $v_{i}$. The purpose of $S_{1}$ is to lift ${ }^{4}$ a vertex $v_{i}(i>2)$ from $\Pi_{x y}$ to $\Pi_{\varepsilon}$. To do this, rotate $v_{i}$ about the line through $v_{i-1}^{\prime}$ and $v_{i+1}$. Thus $v_{i}$ moves on a circle $C$ with center on $v_{i-1}^{\prime} v_{i+1}$. See Fig. 7. Note that circle $C$ might lie partially below $\Pi_{x y}$, and that unless $v_{i}$ is the last vertex, $v_{n-1}$, the plane that contains $C$ is not vertical.

$S_{1}$-constraints: when vertex lifting is applied to a vertex $v_{i}$, for $2<i \leq n-1, \varepsilon$ must

\footnotetext{
${ }^{3}$ We call this the St. Louis Arch Algorithm because of the resemblance to the arch in St. Louis, Missouri.

${ }^{4}$ When referring to Fig. 6, note that the vertex to be lifted next in that figure is $v_{i+1}$.
} 


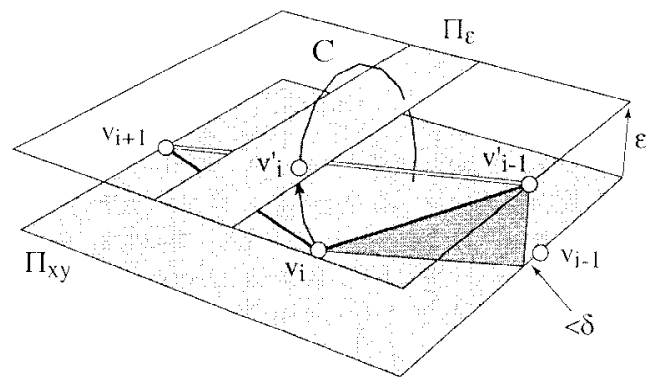

Fig. 7. Vertex $v_{i}$ rotates around $C$ up to $\Pi_{\varepsilon} ; v_{i-1}^{\prime}$ projects to within $\delta$ of $v_{i-1}$.

be chosen so that $C$ intersects $\Pi_{\varepsilon}$, and so that $v_{i}^{\prime}$ projects to $\Pi_{x y}$ no more than $\delta$ away from $v_{i}$ (to satisfy $\mathrm{H} 3$ ).

$S_{2}$ : lowering the arch. After $v_{i}$ has been raised by $S_{1}$, edge $v_{i-1}^{\prime} v_{i}^{\prime}$ lies in $\Pi_{\varepsilon}$. If $\mathrm{H} 2$ holds, $\operatorname{arch} A$ is a convex chain lying (except for its base vertices) above $\Pi_{\varepsilon}$ in $\Pi_{z}\left(v_{0}^{\prime}, v_{i-1}^{\prime}\right)$. Procedure $S_{2}$ now rotates the arch $A$ about the line through $v_{0}^{\prime} v_{i-1}^{\prime}$ away from edge $v_{i-1}^{\prime} v_{i}^{\prime}$ until $A$ reaches plane $\Pi_{\varepsilon}$. It is possible that $\Delta v_{0}^{\prime} v_{i-1}^{\prime} v_{i}^{\prime}$ is degenerate; in any case, the chain $P\left(v_{0}^{\prime}, v_{1}^{\prime}, \ldots, v_{i-2}^{\prime}, v_{i-1}^{\prime}\right)$, without its endpoints, lies strictly to one side of the line through $v_{0}^{\prime} v_{i-1}^{\prime}$. See Fig. 8.

$S_{3}$ : reconvexifying the arch. At the start of $S_{3}$, the arch $A$ lies in $\Pi_{\varepsilon}$, to one side of the line through $v_{0}^{\prime} v_{i-1}^{\prime}$, and $v_{i}^{\prime}$ lies either on this line, or on the opposite side of this line. Vertices $v_{0}^{\prime}$ and $v_{i}^{\prime}$ will become the base points for the new arch $A$ and are held fixed while reconvexification is performed.

If $v_{i}^{\prime}$ lies on the line through the old base points $v_{0}^{\prime}$ and $v_{i-1}^{\prime}$, then $S_{3}$ resolves this

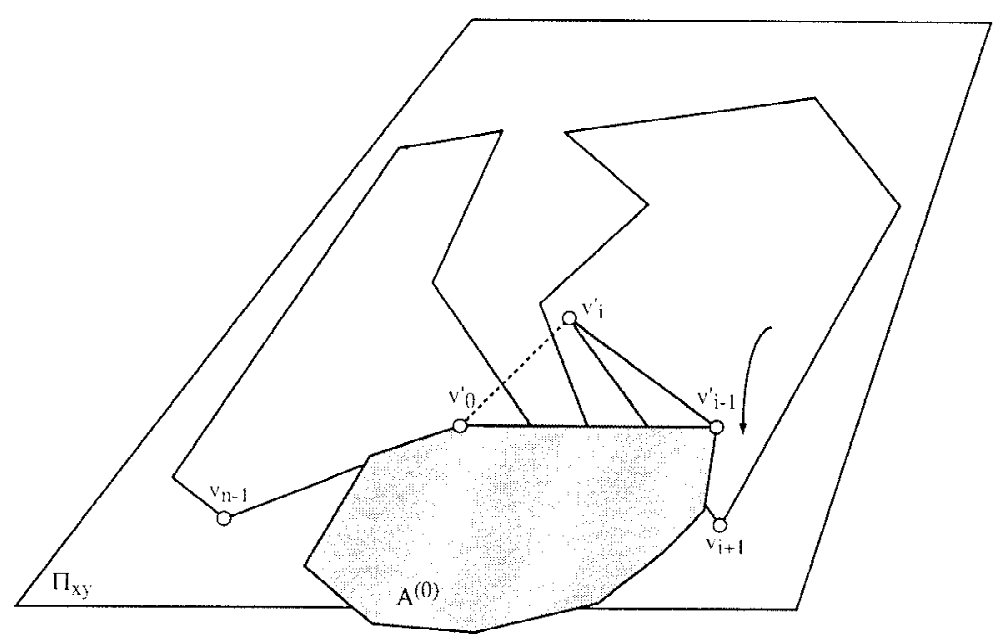

Fig. 8. The arch $A$ and $\Delta v_{0}^{\prime} v_{i-1}^{\prime} v_{i}^{\prime}$ lie in plane $\Pi_{\varepsilon}$ (not shown), floating $\varepsilon$ above $\Pi_{x y}$. 


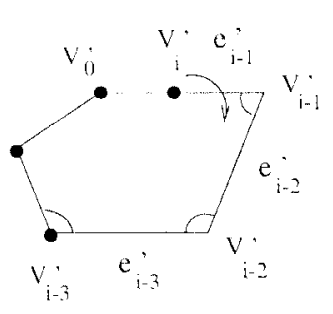

(a)

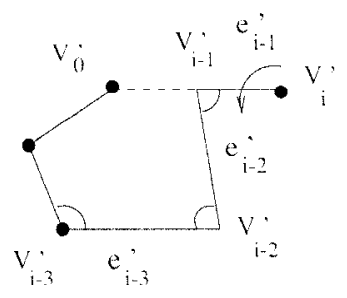

(b)

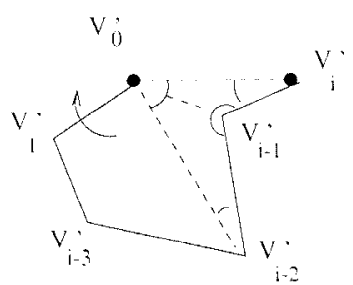

(c)

Fig. 9. (a) $v_{i}^{\prime}$ between $v_{0}^{\prime}$ and $v_{i-1}^{\prime}$; (b) $v_{i-1}^{\prime}$ between $v_{0}^{\prime}$ and $v_{i}^{\prime}$; (c) $v_{i-1}^{\prime}$ is to straighten.

degeneracy as follows. There are two cases, depending on whether $v_{i}^{\prime}$ lies between $v_{0}^{\prime}$ and $v_{i-1}^{\prime}$ or whether $v_{i-1}^{\prime}$ lies between $v_{0}^{\prime}$ and $v_{i}^{\prime}$. (Assuming hypotheses $\mathrm{H} 1-\mathrm{H} 3$ held at the start of this pass through $S_{1}-S_{4}$, and assuming $\delta$ is chosen as described in $\mathrm{H} 3$, it is not possible for $v_{0}^{\prime}$ to lie between $v_{i-1}^{\prime}$ and $v_{i}^{\prime}$.) In both cases, $S_{3}$ rotates edge $e_{i-1}^{\prime}$ about $v_{i}^{\prime}$ (held fixed) in such a way that $v_{i-1}^{\prime}$ moves to the open half-plane containing the other arch vertices. To track the motion of $v_{i-1}^{\prime}$, fix the positions of $v_{0}^{\prime}$ through $v_{i-3}^{\prime}$, and adjust the joint angles at $v_{i-3}^{\prime}, v_{i-2}^{\prime}$, and $v_{i-1}^{\prime}$. Edge $e_{i-3}^{\prime}$ rotates slightly about its fixed endpoint $v_{i-3}^{\prime}$, and vertices $v_{i-1}^{\prime}$ and $v_{i-2}^{\prime}$ change their positions. See Fig. 9, where black dots indicate which vertex positions are fixed and arrows and arcs indicate which angles change.

If $v_{i}^{\prime}$ lies between $v_{0}^{\prime}$ and $v_{i-1}^{\prime}$, as in Fig. 9(a), then the entire polygonal chain $P\left(v_{0}^{\prime}, \ldots, v_{i}^{\prime}\right)$ becomes strictly convex and $S_{3}$ is done.

In the other case, which Fig. 9(b) shows, $v_{i-1}^{\prime}$ lies between $v_{0}^{\prime}$ and $v_{i}^{\prime}$; the subchain $P\left(v_{0}^{\prime}, \ldots, v_{i-1}^{\prime}\right)$ ending at $v_{i-1}^{\prime}$ remains convex after the small rotation of $e_{i-1}^{\prime}$, but $\triangle v_{0}^{\prime}, v_{i-1}^{\prime}, v_{i}^{\prime}$ is no longer degenerate. Adding a phantom edge $v_{0}^{\prime} v_{i}^{\prime}$ to the subchain $P\left(v_{0}^{\prime}, \ldots, v_{i}^{\prime}\right)$ ending at $v_{i}^{\prime}$ creates a simple "barbed" polygon, shown in Fig. 9(c). A "barbed" polygon is one that contains a diagonal (here, $v_{0}^{\prime} v_{i-1}^{\prime}$ ) that splits the polygon into two convex pieces, one of which is a triangle. In general, such a polygon may have two reflex angles, but in our case the polygon has a unique reflex angle at $v_{i-1}^{\prime} . S_{3}$ convexifies this barbed polygon, which can be done by the following simple inductive process. ${ }^{5}$ Keep the base points $v_{0}^{\prime}$ and $v_{i}^{\prime}$ fixed, and keep the subchain $P\left(v_{0}^{\prime}, v_{1}^{\prime}, \ldots, v_{i-2}^{\prime}\right)$ rigid but allow it to rotate as a unit about its endpoint $v_{0}^{\prime}$. Thus the only angles of the barbed polygon that are allowed to change for now are those at $v_{0}^{\prime}, v_{i}^{\prime}, v_{i-1}^{\prime}$, and $v_{i-2}^{\prime}$. The basic idea is to turn the quadrilateral $v_{0}^{\prime}, v_{i}^{\prime}, v_{i-1}^{\prime}, v_{i-2}^{\prime}$ into a triangle by straightening (and then freezing) $v_{i-1}^{\prime}$. If one of the other angles of the barbed polygon is the first to straighten, then we freeze it immediately. If this occurs, as we are about to see, the result is a barbed polygon on fewer vertices with a unique reflex angle at the same vertex, $v_{i-1}^{\prime}$.

Holding $v_{0}^{\prime}$ and $v_{i}^{\prime}$ fixed and subchain $P\left(v_{0}^{\prime}, v_{1}^{\prime}, \ldots, v_{i-2}^{\prime}\right)$ internally rigid, begin to straighten the angle at $v_{i-1}^{\prime}$. This increases the distance between $v_{i}^{\prime}$ and $v_{i-2}^{\prime}$, so angle $\angle v_{i-2}^{\prime} v_{0}^{\prime} v_{i}^{\prime}$ and hence the internal angle of the barbed polygon at $v_{0}^{\prime}$ also increase. It

\footnotetext{
${ }^{5}$ This process appears in the middle of the proof of Lemma 4, page 313, of [Sa], where the process was designed for a different context, and links were allowed to cross. We give our own version here, for completeness.
} 
can be checked by trigonometry and calculus, or by the geometric argument on pages 25-26 of [BDD $\left.{ }^{+} 2\right]$, that the distance between $v_{0}^{\prime}$ and $v_{i-1}^{\prime}$ increases, which implies that angles $\angle v_{0}^{\prime} v_{i}^{\prime} v_{i-1}^{\prime}$ and $\angle v_{0}^{\prime} v_{i-2}^{\prime} v_{i-1}^{\prime}$, and hence angle $\angle v_{i-1}^{\prime} v_{i-2}^{\prime} v_{i-3}^{\prime}$, are increasing. Since we freeze any internal angle of the barbed polygon that straightens, and since we are straightening the angle at $v_{i-1}^{\prime}$, it is easy to check that no collisions of chain segments occur. Since the angle at $v_{i-1}^{\prime}$ is the only internal angle of the barbed polygon that is decreasing, $v_{i-1}^{\prime}$ remains the only possible reflex angle of the polgyon, which therefore remains barbed if some angle other than $v_{i-1}^{\prime}$ is the first to straighten. This process may be continued inductively, without collisions and with the positions of $v_{0}^{\prime}$ and $v_{i}^{\prime}$ fixed, until a convex configuration is reached.

In case the convexification of the barbed polygon results in a polygon with more than two vertices on the line through $v_{0}^{\prime} v_{i}^{\prime}$, then $S_{3}$ rotates $e_{0}^{\prime}$ and/or $e_{i-1}^{\prime}$ a small amount about $v_{0}^{\prime}$ and/or $v_{i}^{\prime}$, respectively. If $e_{0}^{\prime}$ and/or $e_{i}^{\prime}$ is part of a longer edge containing joints that are frozen straight, then the entire frozen edge is rotated. This will restore $\mathrm{H} 2$ when the arch is lifted.

$S_{4}$ : raising the arch. $S_{4}$ rotates the convexified arch with base points $v_{0}^{\prime}$ and $v_{i}^{\prime}$ about the line through these points up into the vertical plane $\Pi_{z}\left(v_{0}^{\prime}, v_{i}^{\prime}\right)$. Because of strict convexity at $v_{0}^{\prime}$ and $v_{i}^{\prime}$, the remaining vertices of the arch move to positions above $\Pi_{\varepsilon}$.

\subsection{Values for $\varepsilon$ and $\delta$}

Here we compute values for $\varepsilon$ and $\delta$ such that the $S_{0}$-constraints will be satisfied and such that, whenever the hypotheses $\mathrm{H} 1-\mathrm{H} 3$ are satisfied at the beginning of a pass through $S_{1}-S_{4}$, the $S_{1}$-constraints on $\varepsilon$ and $\delta$ incurred during that pass will be satisfied.

Let $\alpha_{j}$ be the angle at $v_{j}$ that is less than $\pi$. Also, let $\beta_{j}=\pi-\alpha_{j}$, the deviation from straightness at joint $v_{j}$; so $\beta_{j}>0$ for all $j$ since by assumption, $P$ has no straight joints.

The fact that $P$ is simple and that each $\beta_{j}>0$ guarantees that a $\delta>0$ as described in $\mathrm{H} 3$ exists. It is a straightforward exercise to compute a $\delta$. Our technical report gives a simple formula.

With $\delta$ chosen, let $\sigma_{\min }$ denote the minimum separation $\left|v_{j} v_{k}\right|$ for all positions of $v_{j}$ and $v_{k}$ within their $\delta$ disks, for all distinct $j$ and $k$. Let $\beta_{\text {min }}$ denote the minimum of all $\beta_{j}$ for all positions of $v_{j}$ within their $\delta$ disks. By H3, $\sigma_{\min }$ and $\beta_{\min }$ are positive.

It is a straightforward exercise, based on examination of the double cone determined by the rotation of $v_{i}$ about the line through its neighbors (see Fig. 7), to compute an $\varepsilon$ value from $\sigma_{\min }$ and $\beta_{\min }$ so that the $S_{0}$-constraints are satisfied, and so that the $S_{1}$-constraints are satisfed under the assumption that $\mathrm{H} 1-\mathrm{H} 3$ hold.

\subsection{Correctness and Complexity}

The St. Louis Arch Algorithm first computes a suitable $\varepsilon$ and $\delta$, as in Section 4.3, then performs the initialization process $S_{0}$, and then makes repeated passes through $S_{1}-S_{4}$ until all vertices have been lifted into a convex arch. The preceding discussion shows that, by induction on the number of passes through $S_{1}-S_{4}$, the following theorem holds. 
Theorem. The St. Louis Arch Algorithm convexifies a planar simple polygon.

Only a constant number of moves are used during each execution of procedures $S_{0}, S_{1}, S_{2}$, and $S_{4}$. Each execution of the reconvexification procedure $S_{3}$ can be done with $O(n)$ moves, so the resulting procedure can be accomplished by $O\left(n^{2}\right)$ moves. Jeff Erikson, and Sallee [Sa], observed that the "freezing" of a joint that becomes straight during the reconvexification procedure $S_{3}$ can only be done $n$ times. This implies, by an amortization argument, that the St. Louis Arch Algorithm makes $O(n)$ moves. Moreover, our technical report $\left[\mathrm{BDD}^{+} 2\right]$ argues that $\varepsilon$ and $\delta$ and the entire sequence of moves can be computed in $O(n)$ time in the extended real RAM model of computation.

\section{Open Problems}

Our results suggest several open questions, including:

1. Characterize the unlocked open and closed chains in three dimensions. What is the complexity of deciding whether a chain in three dimensions is unlocked?

2. The algorithm of Section 3.1 applies to chains with simple orthogonal projections to a plane. In what ways can this approach be generalized to other projections?

3. Can an open chain of unit-length links lock in three dimensions? Cantarella and Johnston show in [CJ] that the answer is no if $n \leq 5$.

\section{Acknowledgments}

We thank Jeff Erickson for the amortization argument and Hazel Everett for useful comments. The idea and terminology for the knitting needles example arose in a 1991 conversation with William Lenhart.

\section{References}

[A] C. C. Adams. The Knot Book. Freeman, New York, 1994

$\left[\mathrm{BDD}^{+} 1\right]$ T. Biedl, E. Demaine, M. Demaine, S. Lazard, A. Lubiw, J. O’Rourke, M. Overmars, S. Robbins, I. Streinu, G. Toussaint, and S. Whitesides. Locked and unlocked polygonal chains in 3D. In Proc. 10th ACM-SIAM Symp. Discrete Algorithms, pp. 866-867, Jan. 1999.

$\left[\mathrm{BDD}^{+} 2\right]$ T. Biedl, E. Demaine, M. Demaine, S. Lazard, A. Lubiw, J. O'Rourke, M. Overmars, S. Robbins, I. Streinu, G. Toussaint, and S. Whitesides. Locked and unlocked polygonal chains in 3D. Archived by arXiv.org as arXiv:cs.CG/9910009 8 Oct. 1999 (see http://xxx.lanl.gov/help/general for information on how to access); also appeared as Smith College Technical Report TR060, 1999, 29 pages.

[BGRT] P. Bose, F. Gomez, P. Ramos, and G. T. Toussaint. Drawing nice projections of objects in space. In Graph Drawing (Proc. GD '95), vol. 1027 of Lecture Notes Computer Science, pp. 52-63. Springer-Verlag, Berlin, 1996.

[Ca] J. Canny. The Complexity of Robot Motion Planning. ACM-MIT Press Doctoral Dissertation Award Series. MIT Press, Cambridge, MA, 1987.

[CDR] R. Connelly, E. Demaine, and G. Rote. Straightening polygonal arcs and convexifying polygonal cycles. In Proc. IEEE Symp. Foundations of Computer Science (FOCS), Nov. 12-14, 2000, pp. 432 442.

[CH] G. Crippen and T. Havel. Distance Geometry and Molecular Conformation. Research Studies Press, Letchworth, 1988. 
[CJ] J. Cantarella and H. Johnston. Nontrivial embeddings of polygonal intervals and unknots in 3-space. J. Knot Theory Ramifications, vol. 7, pp. 1027-1039, 1998.

$\left[\mathrm{CKM}^{+}\right]$J. Calvo, D. Krizanc, P. Morin, M. Soss, and G. Toussaint. Convexifying polygons with simple projections. Inform. Process. Lett., to appear.

[CO] R. Cocan and J. O'Rourke. Polygonal chains cannot lock in 4d. In Proc. 11th Canad. Conf. Computational Geometry, 1999. Extended abstract. Full version: LANL/CoRR paper cs.CG/9908005.

[Cr] P. Cromwell. Polyhedra. Cambridge University Press, Cambridge, 1997.

$\left[\mathrm{ELR}^{+}\right]$H. Everett, S. Lazard, S. Robbins, H. Schröder, and S. Whitesides. Convexifying star-shaped polygons. In Proc. 10th Canad. Conf. Computational Geometry, pp. 2-3, 1998.

[Er] P. Erdôs. Problem 3763. Amer. Math. Monthly, vol. 42, p. 627, 1935.

[GN] C. C. Gibson and P. E. Newstead. On the geometry of the planar 4-bar mechanism. Acta Appl. Math., vol. 7, pp. 113-135, 1986.

[Gr] B. Grünbaum. How to convexify a polygon. Geombinatorics, vol. 5, pp. 24-30, July 1995.

[HJW1] J. E. Hopcroft, D. A. Joseph, and S. H. Whitesides. Movement problems for 2-dimensional linkages. SIAM J. Comput., vol. 13, pp. 610-629, 1984.

[HJW2] J. E. Hopcroft, D. A. Joseph, and S. H. Whitesides. On the movement of robotic arms in 2dimensional bounded regions. SIAM J. Comput., vol. 14, pp. 315-333, 1985.

[Hu] K. H. Hunt. Kinematic Geometry of Mechanisms. Oxford University Press, Oxford, 1978.

[Ka] V. Kantabutra. Reaching a point with an unanchored robot arm in a square. Internat. J. Comput. Geom., vol. 7, pp. 539-550, 1997.

[Ko] J. U. Korein. A Geometric Investigation of Reach. ACM Distinguished Dissertations Series. MIT Press, Cambridge, MA, 1985.

[KSW] M. van Kreveld, J. Snoeyink, and S. Whitesides. Folding rulers inside triangles. Discrete Comput. Geom., vol. 15, pp. 265-285, 1996.

[Li] C. Livingston. Knot Theory. The Mathematical Association of America, Washington, DC, 1993.

[LW1] W. J. Lenhart and S. H. Whitesides. Reconfiguration with line tracking motions. In Proc. 4th Canad. Conf. Computational Geometry, pp. 198-203, 1992.

[LW2] W. J. Lenhart and S. H. Whitesides. Reconfiguring closed polygonal chains in Euclidean $d$-space. Discrete Comput. Geom., vol. 13, pp. 123-140, 1995.

[Mc] J. M. McCarthy. Geometric Design of Linkages. Springer-Verlag, New York, 2000.

[Mi] K. Millett. Knotting of regular polygons in 3-space. J. Knot Theory Ramifications, vol. 3, 1994, pp. 263-278.

[MS] N. Madras and G. Slade. The Self-Avoiding Walk. Birkhäuser, Boston, 1993.

[Na] B. de Sz. Nagy. Solution to problem 3763. Amer. Math. Monthly, vol. 46, pp. 176-177, 1939

[Sa] G. T. Sallee. Stretching chords of space curves. Geom. Dedicata, vol. 2, pp. 311-315, 1973.

[SS] J. T. Schwartz and M. Sharir. On the "piano movers" problem, II: General techniques for computing topological properties of real algebraic manifolds. Adv. Appl. Math., vol. 4, pp. 298-351, 1983.

[St] I. Streinu. A combinatorial approach to planar non-colliding robot arm motion planning. In Proc. IEEE Symp. Foundations of Computer Science (FOCS), Nov. 12-14, 2000, pp. 443-453.

[To1] G. T. Toussaint. The Erdős-Nagy theorem and its ramifications. In Proc. 11th Canad. Conf. Computational Geometry, 1999. Extended abstract.

[To2] G. T. Toussaint. A new class of stuck unknots in Pol-6. Beiträge Algebra Geom. (also known as Contrib. Algebra Geom.), to appear, 2001.

[Wh1] S. H. Whitesides. Algorithmic issues in the geometry of planar linkage movement. Austral. Comput. J., vol. 24, pp. 42-50, 1992.

[Wh2] W. Whiteley. Rigidity and scene analysis. In J. E. Goodman and J. O'Rourke, editors, Handbook of Discrete and Computational Geometry, chapter 49, pp. 893-916. CRC Press, Boca Raton, FL, 1997.

Received October 9, 1999, and in revised form February 6, 2001, and April 26, 2001.

Online publication August 28, 2001. 